\title{
PRIME IDEALS IN SKEW LAURENT POLYNOMIAL RINGS
}

\author{
by K. W. MACKENZIE
}

(Received 2nd August 1991)

Let $R$ be a commutative ring and $\left\{\sigma_{1}, \ldots, \sigma_{n}\right\}$ a set of commuting automorphisms of $R$. Let $T=$ $R\left[\theta_{1}^{ \pm 1}, \ldots, \theta_{n}^{ \pm 1} ; \sigma_{1}, \ldots, \sigma_{n}\right]$ be the skew Laurent polynomial ring in $n$ indeterminates over $R$ and let $S=R\left[x_{1}^{ \pm 1}, \ldots, x_{n}^{ \pm 1}\right]$ be the Laurent polynomial ring in $n$ central indeterminates over $R$. There is an isomorphism $\phi$ of right $R$-modules between $T$ and $S$ given by $\phi\left(\theta_{j}\right)=x_{j}$. We will show that the map $\phi$ induces a bijection between the prime ideals of $T$ and the $\Gamma$-prime ideals of $S$, where $\Gamma$ is a certain set of endomorphisms of the $\mathbb{Z}$-module $S$. We can study the structure of the lattice of $\Gamma$-prime ideals of the ring $S$ by using commutative algebra, and this allows us to deduce results about the prime ideal structure of the ring $T$. As an example, if $R$ is a Cohen-Macaulay $\mathbb{C}$-algebra and the action of the $\sigma_{j}$ on $R$ is locally finite-dimensional, we will show that the ring $T$ is catenary.

1991 Mathematics subject classification: 16D30, 16P40, 16S34, 16 S36.

\section{Introduction}

In this paper we will investigate the prime ideal structure of a skew Laurent polynomial extension

$$
T=R\left[\theta_{1}, \theta_{1}^{-1}, \theta_{2}, \theta_{2}^{-1}, \ldots, \theta_{n}, \theta_{n}^{-1} ; \sigma_{1}, \sigma_{2}, \ldots, \sigma_{n}\right]
$$

where $R$ is a ring and the $\sigma_{j}$ are commuting automorphisms of $R$. We obtain fairly detailed results if $R$ is a commutative ring and the $\sigma_{j}$ satisfy appropriate conditions; for instance, if $R$ is an affine $\mathbb{C}$-algebra and the action of the $\sigma_{j}$ on $R$ is locally finite-dimensional then we will show that $T$ is a catenary ring.

Much of the work here is based on [3], where the case of an extension involving derivations is treated. Related results are also contained in [2].

Acknowledgments. The research for this paper was carried out at the University of Edinburgh with the financial support of the Science and Engineering Research Council. I would like to thank my Ph.D. supervisor, Dr. T. H. Lenagan, for his help and advice during the course of my studies.

\section{Ideals in skew Laurent polynomial rings}

1.1. The following notation will be used throughout. 
Notation. $\quad k$ is a commutative ring and $R$ an associative $k$-algebra. $\Sigma$ denotes a finite sequence $\left(\sigma_{1}, \ldots, \sigma_{n}\right)$ of (not necessarily distinct) $k$-automorphisms of $R$, and $G=$ $\left\langle\sigma_{1}, \ldots, \sigma_{n}\right\rangle \leqq \mathrm{Aut}_{k} R$ is the subgroup of $\mathrm{Aut}_{k} R$ generated by the $\sigma_{j}$. We will always suppose that the $\sigma_{i}$ commute, so that $G$ is abelian. Let

$$
T=R\left[\theta_{1}, \theta_{1}^{-1}, \theta_{2}, \theta_{2}^{-1}, \ldots, \theta_{n}, \theta_{n}^{-1} ; \sigma_{1}, \sigma_{2}, \ldots, \sigma_{n}\right]
$$

be the ring of skew Laurent polynomials over $R$ (see $[8, \$ 1.2,1.4,1.6]$ for general properties of such rings). We will view $T$ as a ring of Laurent polynomials in the $\theta_{j}$ with coefficients from $R$ written on the right; thus an element $t \in T$ can be written uniquely in the form

$$
\sum_{I \in \mathbf{Z}^{n}} \theta^{I} r_{I}
$$

where $r_{I} \in R$ and $r_{I}=0$ for all but finitely many $I \in \mathbb{Z}^{n}$, and $\theta^{I}=\theta_{n}^{i_{n}} \ldots \theta_{1}^{i_{1}}$ for $I=$ $\left(i_{1}, \ldots, i_{n}\right) \in \mathbb{Z}^{n}$ (note the reversal of the order of the $i_{k}$ ).

The additive structure of $T$ is the same as that of the usual Laurent polynomial ring. Elements of $R$ are multiplied together in the normal way, and the $\theta_{j}$ commute with each other, but we have

$$
r \theta_{j}=\theta_{j} \sigma_{j}(r) \text { for } \quad r \in R \text {. }
$$

Since $\theta_{j}$ is a unit in $T$ the above equation can be written in the form $\sigma_{j}(r)=\theta_{j}^{-1} r \theta_{j}$; thus the automorphism $\sigma_{j}$ becomes an inner automorphism in $T$. If we use the symbol " $\sigma^{I}$ " to denote $\sigma_{n}^{i_{n}} \ldots \sigma_{1}^{i_{1}}$ then (using the fact that that the $\sigma_{j}$ commute) we see that $r \theta^{I}=\theta^{I} \sigma^{I}(r)$ for all $r \in R, I \in \mathbb{Z}^{n}$.

Let

$$
S=R\left[x_{1}, x_{1}^{-1}, \ldots, x_{n}, x_{n}^{-1}\right]
$$

be the Laurent polynomial ring in $n$ central indeterminates $x_{1}, \ldots, x_{n}$; we will write elements of $S$ using a similar multi-index notation to that above.

We will occasionally need to consider the skew polynomial rings $S^{+}=R\left[x_{1}, \ldots, x_{n}\right]$ and $T^{+}=R\left[\theta_{1}, \ldots, \theta_{n} ; \sigma_{1}, \ldots, \sigma_{n}\right]$. The ring $T^{+}$has a similar structure to that of $T$; the elements $\theta_{j}$ are no longer units of $T^{+}$, but it can be seen with the aid of (*) that each $\theta_{j}$ is a normal element of $T^{+}$(i.e., $\theta_{j} T^{+}=T^{+} \theta_{j}$ for all $j$ ). We will view $T^{+}$as being a subring of $T$ in the natural way; we may then regard $T$ as being the localisation of $T^{+}$ at the Ore set generated by $\left\{\theta_{1}, \ldots, \theta_{n}\right\}$; similarly we will regard $S^{+}$as a subring of $S$ and $S$ as a localisation of $S^{+}$.

Note that if $R$ is a Noetherian ring then so is each of the rings $T, T^{+}, S$ and $S^{+}$: see $[8,1.2 .9(i v)]$.

1.2. We wish to study the relationship between the ideals of $T$ and those of $S$. We 
will define a map $\phi$ from $T$ to $S$ and we will show that under this map the prime ideals of $T$ are in one-to-one correspondence with the $\Gamma$-prime ideals of $S$, where $\Gamma \supseteq G$ is a certain subset of $\operatorname{End}_{k} S$. Firstly we require some standard definitions and some notation.

Definition. Let $A$ be a ring and $\Gamma$ a subset of $\operatorname{End}_{\mathrm{z}} A$. If $\gamma \in \Gamma$ then we will denote the action of $\gamma$ on $A$ by $a \mapsto a^{\gamma}(a \in A)$. If $X \subseteq A$ then $X^{\gamma}=\left\{x^{\gamma}: x \in X\right\}$. We say that $K \subseteq A$ is a right $\Gamma$-ideal of $A$ if $K$ is a right ideal of $A$ and $K$ is $\Gamma$-stable (i.e., $K^{\gamma} \subseteq K$ for all $\gamma \in \Gamma$ ). We denote the lattice of right $\Gamma$-ideals of $A$ by $\mathscr{L}_{\Gamma}(A)$. If $I \unlhd A$ then we say that $I$ is a $\Gamma$-ideal of $A$, and write $I \unlhd_{\Gamma} A$, if $I^{\nu} \subseteq I$ for all $\gamma \in \Gamma$. A proper ideal $Q$ of $A$ is a $\Gamma$-prime ideal of $A$ if $Q \unlhd_{\mathrm{r}} A$ and the following holds:

$$
I, J \unlhd_{\Gamma} A \text { and } I J \subseteq Q \Rightarrow I \subseteq Q \text { or } J \subseteq Q .
$$

The set of $\Gamma$-prime ideals of $A$ will be denoted by $\operatorname{Spec}^{\Gamma}(A)$. If $I \unlhd A$ then we write $I^{\Gamma}=\cap\left\{I^{\gamma}: \gamma \in \Gamma\right\}$ : it is clear that if $\Gamma$ is a group of automorphisms of $A$ then $I^{\Gamma}$ is the largest $\Gamma$-ideal contained in $I$, and that in this case if $P \in \operatorname{Spec} A$ then $P^{\Gamma} \in \operatorname{Spec}^{\Gamma} A$.

1.3. A special case that will arise later occurs when $A$ is Noetherian and $\Gamma$ is a subgroup of Aut $A$; then $\Gamma$ acts naturally on $\operatorname{Spec} A$ (for if $P \in \operatorname{Spec} A$ and $\gamma \in \Gamma$ then it is easy to see that $P^{\gamma} \in \operatorname{Spec} A$ ) and there is a well-known result giving a precise description of $\operatorname{Spec}^{\Gamma} A$.

Theorem. Suppose that $A$ is a Noetherian ring and $H$ is a group of automorphisms of $A$. Let $Q \in \operatorname{Spec}^{H} A$ and let $\Omega$ be the (finite) set of primes minimal over $Q$. Then $\Omega$ forms $a$ complete orbit for the action of $H$ on $\operatorname{Spec} A$, and

$$
\begin{aligned}
Q & =P^{H} \text { for all } P \in \Omega \\
& =P^{h_{1}} \cap \cdots \cap P^{h_{n}} \text { for some } h_{j} \in H .
\end{aligned}
$$

Thus every $H$-prime ideal is semiprime.

Proof. See [9, Lemma 5], for example.

Note that it is possible to have $Q=P^{H}$ with $P$ not minimal over $Q$ : for instance if $A=\mathbb{Q}[x]$ and $H$ is the subgroup of Aut $A$ generated by $x \mapsto x+1$ then $0 \in \operatorname{Spec}^{H} A$ and $0=0^{H}=(x)^{H}$.

1.4. We can now return to the skew Laurent polynomial ring $T$. The result below is very simple but it will turn out to be extremely useful.

Lemma. Every two-sided ideal of $T$ is G-stable (where $G$ is the subgroup of $\mathrm{Aut}_{k} T$ generated by $\left.\left\{\sigma_{1}, \ldots, \sigma_{n}\right\}\right)$; in other words, $J \unlhd T \Rightarrow J \in \mathscr{L}_{G}(T)$. 
Proof. Let $J$ be a two-sided ideal of $T$. We have $\sigma_{j}(J)=\theta_{j}^{-1} J \theta_{j}$ and since $\theta_{j}$ is a unit in $T, \theta_{j}^{-1} J \theta_{j}=J$ for all $j$; it follows that $J$ is $G$-stable.

1.5. The rings $T$ and $S$ are free right $R$-modules with bases of the same cardinality and are thus isomorphic as right $R$-modules; we will use this isomorphism to relate the ideal structure of $T$ to that of $S$.

Definition. Let $R, T$ and $S$ be as in 1.1. Define $\phi: T \rightarrow S$ by

$$
\phi\left(\sum \theta^{I} r_{l}\right)=\sum x^{I} r_{I}
$$

$\phi$ is clearly an isomorphism of right $R$-modules and

$$
\phi\left(\theta^{I} t r\right)=x^{I} \phi(t) r=\phi(t) x^{I} r \quad \text { for all } I \in \mathbb{Z}^{n}, r \in R, t \in T .
$$

Moreover, if we extend the $\sigma_{j}$ to $T$ and $S$ in the obvious manner (i.e., so that they act trivially on the indeterminates) then $\phi \sigma_{j}=\sigma_{j} \phi$ for all $j$. Note that $\phi$ restricts to a map $T^{+} \rightarrow S^{+}$: we will denote this map by $\phi$ also.

Proposition 1.6. $\phi$ induces an isomorphism between $\mathscr{L}_{G}(T)$ and $\mathscr{L}_{G}(S)$; this restricts to an isomorphism between $\mathscr{L}_{G}\left(T^{+}\right)$and $\mathscr{L}_{G}\left(S^{+}\right)$.

Proof. Let $K \in \mathscr{L}_{G}(T)$; then $\phi(K) \leqq S_{R}$. Also,

$$
\sigma^{I} \phi(K)=\phi \sigma^{I}(K) \subseteq \phi(K) \quad \forall I \in \mathbb{Z}^{n}
$$

in other words, $\phi(K)$ is $G$-stable. Thus

$$
\begin{aligned}
\phi(K) \cdot \sum x^{I} r_{I} & =\sum x^{I} \phi(K) r_{I} \\
& =\phi\left(\sum \theta^{I} K r_{I}\right) \\
& =\phi\left(\sum \sigma^{-I}(K) \theta^{I} r_{I}\right) \\
& \subseteq \phi\left(\sum K \theta^{I} r_{I}\right) \\
& \subseteq \phi(K),
\end{aligned}
$$

so $\phi(K) \leqq S_{S}$. Thus $\phi(K) \in \mathscr{L}_{G}(S)$.

Similarly, if $L \in \mathscr{L}_{G}(S)$ then $\sigma^{I} \phi^{-1}(L)=\phi^{-1} \sigma^{I}(L) \subseteq \phi^{-1}(L)$ and hence

$$
\begin{aligned}
\phi^{-1}(L) \cdot \sum \theta^{I} r_{I} & =\sum \theta^{I} \sigma^{I} \phi^{-1}(L) r_{I} \\
& \subseteq \sum \theta^{I} \phi^{-1}(L) r_{I}
\end{aligned}
$$




$$
\begin{aligned}
& =\phi^{-1}\left(\sum x^{I} L r_{1}\right) \\
& \subseteq \phi^{-1}(L)
\end{aligned}
$$

so $\phi^{-1} L \in \mathscr{L}_{G}(T)$.

Thus $\phi$ induces a bijection between $\mathscr{L}_{G}(T)$ and $\mathscr{L}_{G}(S)$, and this map is clearly an isomorphism of lattices.

The same argument shows that the restriction of $\phi$ to $T^{+}$induces an isomorphism $\mathscr{L}_{G}\left(T^{+}\right) \rightarrow \mathscr{L}_{G}\left(S^{+}\right)$

1.7. We wish to find out what happens to two-sided ideals of $T$ under $\phi$. It turns out that we need some more endomorphisms to do this.

Definition. If $r \in R$ define $\chi_{r}: S \rightarrow S$ by

$$
\chi_{r}(s)=\phi\left(r \phi^{-1}(s)\right) \quad(s \in S) .
$$

$\chi_{r}$ is the map of $S$ induced by left multiplication by $r$ in $T$.

Lemma 1.8. If $r \in R$ then

(i) $\chi_{r}\left(\sum x^{I} a_{I}\right)=\sum x^{I} \sigma^{I}(r) a_{I}$

(ii) $\chi_{r}(a)=r a \quad \forall a \in R$

(iii) $\chi_{r}(s a)=\chi_{r}(s) a \quad \forall s \in S, a \in R$

(iv) $x^{I} \cdot \chi_{r}(s)=\chi_{a-1(r)}\left(x^{I} s\right) \quad \forall s \in S, I \in \mathbb{Z}^{n}$

(v) $\chi_{r}\left(x^{I}\right)=x^{I} \sigma^{I}(r) \quad \forall I \in \mathbb{Z}^{n}$.

Proof. These facts are easy consequences of the definition of the $\chi_{r}$.

Note that by (iii), $\chi_{\mathrm{r}} \in \operatorname{End}_{R}\left(S_{R}\right)$. If we define $\chi: R \rightarrow \operatorname{End}_{R}\left(S_{R}\right)$ by $\chi(r)=\chi_{\mathrm{r}}$ then it can be shown that $\chi$ is an injective homomorphism of rings.

1.9. We can now show that the ideals of $T$ correspond to those right ideals of $S$ which are stable both under $G$ and under each $\chi_{r}$.

\section{Definition.}

$$
\Gamma:=G \cup \chi(R) \subseteq \operatorname{End}_{k} S
$$

Proposition 1.10. $\phi$ induces an isomorphism between the lattice of two-sided ideals of $T$ and the lattice of $\Gamma$-stable right ideals of $S$.

Proof. Firstly, recall from Lemma 1.4 that all two-sided ideals of $T$ are $G$-stable; thus if $A \unlhd T$, Lemma 1.6 shows that $\phi(A) \in \mathscr{L}_{G}(S)$, so we need only show that $\phi(A)$ is 
$\chi_{r}$-stable for each $r$ in $R$; this follows because $\chi_{r} \phi(A)=\phi\left(r \phi^{-1} \phi(A)\right)=\phi(r A) \subseteq \phi(A)$ for all $r \in R$.

Conversely, let $K$ be a $\Gamma$-stable right ideal of $S$. Then $\phi^{-1} K$ is a $G$-stable right ideal of $T$. We have $\theta^{I} \phi^{-1} K=\phi^{-1}\left(x^{I} K\right)=\phi^{-1}\left(K x^{I}\right) \subseteq \phi^{-1} K$; also, if $r \in R$ then $r \phi^{-1}(K)=$ $\phi^{-1}\left(\phi\left(r \phi^{-1}(K)\right)\right)=\phi^{-1}\left(\chi_{r}(K)\right) \subseteq \phi^{-1}(K)$. It follows that $T \phi^{-1}(K) \subseteq \phi^{-1}(K)$ and hence $\phi^{-1}(K) \unlhd T$.

Note. The same proof shows that $\phi$ induces an isomorphism between the lattice of $G$-stable two-sided ideals of $T^{+}$and the lattice of $\Gamma$-stable right ideals of $S^{+}$.

\section{Prime ideals in skew Laurent polynomial rings}

2.1. From now on we will assume that $R$ is a commutative ring, so that $S$ is also commutative. Proposition 1.10 now reads as follows:

Proposition. If $R$ is a commutative ring then the map $\phi$ induces an isomorphism between the lattice of two-sided ideals of $T$ and the lattice of $\Gamma$-stable ideals of $S$.

This result will allow us to investigate the ideals of $T$ by using commutative algebra.

2.2. Our objective in this section is to show that the prime ideals of $T$ correspond to the $\Gamma$-prime ideals of $S$. In rings of polynomials, results can often be proved by induction on the degree of an element. In the rings of Laurent polynomials considered here it is convenient to use a different measure of the size of a polynomial.

Definition. If $t=\sum \theta^{I} r_{I} \in T$ then the support of $t$ is $\operatorname{supp}(t)=\left\{I \in \mathbb{Z}^{n}: r_{I} \neq 0\right\}$, and the width of $t$ is width $(t)=|\operatorname{supp}(t)|($ note that width $(t)<\infty$ for all $t \in T$ ). The constant term of $t$ is $r_{0}$. We make similar definitions in $S$.

Thus if $t=\theta^{-7}+8+\theta^{19} \in R\left[\theta, \theta^{-1} ; \sigma\right]$ then the width of $t$ is 3 (not 26) and the constant term of $t$ is 8 .

2.3. In the following results, the symbol " $[x, y]$ " denotes $x y-y x$; if $X$ and $Y$ are subsets of some ring then $[X, Y]=\{[x, y]: x \in X, y \in Y\}$.

Lemma. If $r \in R$ and $t \in T$ then width $([r, t]) \leqq$ width $(t)$. If the constant term of $t$ is nonzero then width $([r, t])<$ width $(t)$.

Proof. If $t=\sum \theta^{I} a_{I} \in T$ and $r \in R$ then a simple calculation shows that $[r, t]=\sum \theta^{I} b_{I}$, where $b_{I}=\sigma^{I}(r) a_{I}-a_{I} r$ (note that $b_{0}=0$ ), and the result follows easily.

Lemma 2.4. Let $A$ be an ideal of $T$ and suppose that $c \in T$ is such that $[R, c] \subseteq A$. If $t \in T$ then $\phi(t c)-\phi(t) \phi(c) \in \phi(A)$.

Proof. Put $t=\sum \theta^{I} r_{I}$. Then 


$$
\begin{aligned}
\phi(t c)-\phi(t) \phi(c) & =\phi\left(\sum \theta^{I} r_{I} c\right)-\phi\left(\sum \theta^{I} r_{I}\right) \phi(c) \\
& =\phi\left(\sum \theta^{I}\left[r_{I}, c\right]+\sum \theta^{I} c r_{I}\right)-\sum x^{I} r_{I} \phi(c) \\
& =\phi\left(\sum \theta^{I}\left[r_{I}, c\right]\right)+\sum x^{I} \phi(c) r_{I}-\sum x^{I} r_{I} \phi(c) \\
& =\phi\left(\sum \theta^{I}\left[r_{I}, c\right]\right), \text { since } R \text { is commutative } \\
& \in \phi(A), \text { since }[R, c] \subseteq A .
\end{aligned}
$$

Lemma 2.5. Let $A$ be an ideal of $T$. If $C$ is a $G$-stable subset of $T$ such that $[R, C] \subseteq A$ then $T C+A=C T+A$ is a two-sided ideal of $T$.

Proof. If $t=\sum \theta^{I} r_{I} \in T$ and $c \in C$ then $t c=\sum \theta^{I} r_{I} c=\sum \theta^{I}\left[r_{l}, c\right]+\sum \theta^{I} c r_{I}=\sum \theta^{I}\left[r_{l}, c\right]+$ $\sum \sigma^{-I}(c) \theta^{I} r_{I} \in A+C T$, since $[R, c] \subseteq A$ and $\sigma^{-I}(c) \in C$ for all $I \in \mathbb{Z}^{n}$. Thus $T C \subseteq A+C T$. A similar argument shows that $C T \subseteq T C+A$, and the result follows.

Lemma 2.6. Let $A$ be an ideal of $T$ and let $C$ be $a$ G-stable subset of $T$ with $[R, C] \subseteq A$. If we put $\tilde{C}=A+T C$ then $\tilde{C}$ is an ideal of $T$, and if $K$ is any right ideal of $T$ we have $\phi(K \tilde{C})+\phi(A)=\phi(K) \phi(\tilde{C})+\phi(A)$.

Proof. By Lemma $2.5, \tilde{C}$ is an ideal of $T$. Lemma 2.4 shows that $\phi(t c)-$ $\phi(t) \phi(c) \in \phi(A)$ for every $t \in T$ and $c \in C$; it follows that $\phi(K C)+\phi(A)=\phi(K) \phi(C)+\phi(A)$ for any right ideal $K$ of $T$. Now

$$
\begin{aligned}
\phi(K \tilde{C}) & =\phi(K(A+T C)) \\
& \subseteq \phi(K A+K T C) \\
& \subseteq \phi(A+K C) \\
& =\phi(A)+\phi(K C) \\
& =\phi(A)+\phi(K) \phi(C) \\
& \subseteq \phi(A)+\phi(K) \phi(\tilde{C}) .
\end{aligned}
$$

Also,

$$
\begin{aligned}
\phi(K) \phi(\tilde{C}) & =\phi(K)(\phi(T C)+\phi(A)) \\
& \subseteq \phi(K) \phi(T C)+\phi(A) \\
& \subseteq \phi(K)(\phi(T) \phi(C)+\phi(A))+\phi(A), \text { by Lemma } 2.4
\end{aligned}
$$




$$
\begin{aligned}
& =\phi(K) \phi(T) \phi(C)+\phi(A) \\
& \subseteq \phi(K) \phi(C)+\phi(A) \\
& =\phi(K C)+\phi(A), \text { by Lemma } 2.4 \\
& \subseteq \phi(K \tilde{C})+\phi(A) .
\end{aligned}
$$

Thus $\phi(K \tilde{C})+\phi(A)=\phi(K) \phi(\tilde{C})+\phi(A)$, as claimed.

Theorem 2.7. Suppose that $A$ is an ideal of $T$. If $A<F \unlhd T$ then there exists $F^{\prime} \unlhd T$ with $A<F^{\prime} \leqq F$ such that $\phi\left(K F^{\prime}\right)+\phi(A)=\phi(K) \phi\left(F^{\prime}\right)+\phi(A)$ for any right ideal $K$ of $T$.

Proof. Let $w=\operatorname{Min}\{$ width $(f): f \in F \backslash A\}$ and let $C=\left\{c \in F\right.$ : width $(c)=w$ and $\left.c_{0} \neq 0\right\}$. Note that $C \neq \varnothing$, since having found $f \in F \backslash A$ with width $(f)=w$, we have $\theta^{I} f \in F \backslash A$ for all $I \in \mathbb{Z}^{n}$, and $\theta^{I} f$ will have a nonzero constant term for an appropriate value of $I$; in this case we in fact have $\theta^{I} f \in C \backslash A$. Now $C$ is clearly a $G$-stable subset of $T$, and if $r \in R$ and $c \in C$ then 2.3 shows that width $([r, c])<$ width $(c)=w$; since $[r, c] \in F$, the choice of $w$ means that $[r, c] \subseteq A$, so that $[R, C] \subseteq A$. Lemma 2.6 now shows that $F^{\prime}:=A+T C$ behaves as required.

Theorem 2.8. $\phi$ induces a bijection between $\operatorname{Spec} T$ and $\operatorname{Spec}^{\Gamma} S$.

Proof. Suppose that $B \in \operatorname{Spec}^{\Gamma} S$ and put $A=\phi^{-1} B$, which is a two-sided ideal of $T$ by Proposition 2.1. Let $E$ and $F$ be ideals of $T$ with $A \subseteq E \cap F$ and $E F \subseteq A$. If $F \nsubseteq A$, construct $F^{\prime} \unlhd T$ with $A<F^{\prime} \leqq F$ as in Theorem 2.7. Now $\phi(E)$ and $\phi\left(F^{\prime}\right)$ are both $\Gamma$-ideals of $S$, and $\phi(E) \phi\left(F^{\prime}\right) \subseteq \phi\left(E F^{\prime}\right)+\phi(A)=B$ since $E F^{\prime} \subseteq E F \subseteq A$. Since $B$ is a $\Gamma$ prime ideal of $S$ and $\phi\left(F^{\prime}\right) \nsubseteq B$, we have $\phi(E) \subseteq B=\phi(A)$ and so $E \subseteq A$. Thus $A$ is a prime ideal of $T$.

Now suppose that $A$ is a prime ideal of $T$ and put $B=\phi(A) \unlhd_{\Gamma} S$. Suppose that $U$ and $V$ are $\Gamma$-ideals of $S$ with $B \subseteq U \cap V$ and $U V \subseteq B$. Put $E=\phi^{-1}(U)$ and $F=\phi^{-1}(V)$, and note that $E$ and $F$ are two-sided ideals of $T$. If $V \nsubseteq B$ then $A<F$ and Theorem 2.7 again provides us with an ideal $F^{\prime}$ of $T$ such that $A<F^{\prime} \leqq F$ and $\phi\left(E F^{\prime}\right)+\phi(A)=$ $\phi(E) \phi\left(F^{\prime}\right)+\phi(A)$. Now $\phi\left(E F^{\prime}\right) \subseteq \phi(E) \phi\left(F^{\prime}\right)+\phi(A) \subseteq \phi(E) \phi(F)+B=U V+B=B=\phi(A)$. Therefore $E F^{\prime} \subseteq A$ and since $F^{\prime} \nsubseteq A$ we have $E \subseteq A$ and hence $U=\phi(E) \subseteq \phi(A)=B$. It follows that $B$ is a $\Gamma$-prime ideal of $S$.

2.9. We require one more result in this section.

Lemma. If $B \in \operatorname{Spec}^{\Gamma} S$ and $B<V \unlhd_{\Gamma} S$ then $\operatorname{Ann}_{S / B}(V / B)=0$.

Proof. Put $A=\phi^{-1} B$ and $F=\phi^{-1} V$; then $A \in \operatorname{Spec} T$ and $A<F \unlhd T$. Construct an 
ideal $F^{\prime}$ as in 2.7 ; since $F^{\prime}$ is a two-sided ideal of $T$ and $A$ is a prime ideal of $T$, $\operatorname{Ann}_{T / A}\left(F^{\prime} / A\right)=0$. Now

$$
\begin{aligned}
s \in S \text { and } s V \subseteq B & \Rightarrow s \phi\left(F^{\prime}\right) \subseteq B \\
& \Rightarrow s S \phi\left(F^{\prime}\right) \subseteq \phi(A) \\
& \Rightarrow \phi^{-1}(s S) F^{\prime} \subseteq A \text { by Theorem } 2.7 \\
& \Rightarrow \phi^{-1}(s S) \subseteq A \\
& \Rightarrow s \in B .
\end{aligned}
$$

Corollary 2.10. Suppose that $R$ is a Noetherian ring. If $Q \in \operatorname{Spec}^{\Gamma} S$ and $H \in \operatorname{Spec} S$ is minimal over $Q$ then $Q$ is the largest $\Gamma$-ideal of $S$ which is contained in $H$.

Proof. $H / Q$ is a minimal prime in the commutative Noetherian ring $S / Q$ and so has nonzero annihilator. It follows from Lemma 2.9 that $H / Q$ contains no nonzero $\Gamma$-ideals of $S / Q$.

\section{Eigenvectors and normal elements}

3.1. Throughout this section we will assume that $R$ is a commutative algebra over a field $k$. We will show that (with suitable restrictions on the action of $G$ on $R$ ), every factor $B / A$ with $A<B \unlhd T$ contains a $G$-eigenvector, and that this eigenvector is a normal element of the ring $T / A$. This will allow us to use the Principal Ideal Theorem to get a grip on the heights of prime ideals of $T$, and to show that $T$ is sometimes catenary. The condition required on $G$ is as follows.

Definition 3.2. Let $A$ be a $k$-algebra, where $k$ is a field, and let $H$ be a subgroup of Aut $_{k} A$. The action of $H$ on $A$ is said to be locally finite-dimensional if every element of $A$ is contained in a finite-dimensional $H$-stable $k$-subspace of $A$.

Examples 3.3. (i) It is easy to see that any finite group of automorphisms of a ring $A$ has a locally finite-dimensional action: if $H=\left\{h_{1}, \ldots, h_{r}\right\}$ and $a \in A$ then $a$ is contained in the $H$-stable subspace $k a^{h_{1}}+k a^{h_{2}}+\cdots+k a^{h_{r}}$, which clearly has dimension at most $r$ over $k$.

(ii) Let $\sigma$ be an automorphism of a commutative polynomial algebra $A=k[x]$. If $\sigma$ satisfies

$$
a \in A \Rightarrow \operatorname{deg} \sigma(a) \leqq \operatorname{deg} a,
$$

then if $a$ is an element of degree $m$ we have 


$$
\left\{\sigma^{n}(a): n \in \mathbb{Z}\right\} \subseteq k \oplus k x \oplus k x^{2} \oplus \cdots \oplus k x^{m},
$$

a finite dimensional $\sigma$-stable subspace of $A$. It follows that any group of automorphisms of $k[x]$ which is generated by degree-preserving automorphisms has a locally finitedimensional action. A similar statement is true for polynomial algebras in several variables, where we consider automorphisms which preserve total degree.

3.4. The following well-known result allows us to construct $G$-eigenvectors in $R$ when the action of $G$ on $R$ is locally finite-dimensional.

Lemma. If $V$ is a finite-dimensional vector space over an algebraically closed field $k$ and $H$ is a finitely generated abelian subgroup of $\operatorname{End}_{k} V$ then $V$ contains a (nonzero) $H$-eigenvector. In other words, there exists $v \in V \backslash 0$ such that $\tau v \in k v$ for all $\tau \in H$.

Proof. The result can be deduced from [4, Proposition 15.4], for example.

3.5. We wish to show that if the action of $G$ on $T$ is locally finite-dimensional then $T$ is "polynormal", in an appropriate sense.

Definition. An element $x$ of a ring $A$ is normal if $x A=A x$. We will say that a ring $A$ is polynormal (respectively, polycentral) if, whenever $I<J$ are two-sided ideals of $A$, there exists $x \in J \backslash I$ such that $x+I$ is a normal (respectively, central) element of the ring $A / I$ (note that $x A+I$ is then a two-sided ideal of $A$ ). We say that a ring is normally separated (respectively, centrally separated) if the condition above holds for all pairs of prime ideals $I<J$.

3.6. We must carry out some explicit calculations in $T$ and $T^{+}$, and we need a fine measure of degree in order to do this.

Definition. Let $n \geqq 1$ and let $I \in \mathbb{Z}^{n}$. The norm of $I$ is $\|I\|=\left|i_{1}\right|+\cdots+\left|i_{n}\right|$. The Dixmier ordering on $\mathbb{Z}^{n}$ is defined as follows:

If $I, J \in \mathbb{Z}^{n}$ then $I<J$ if either

(i) $\|I\|<\|J\|$

or (ii) $\|I\|=\|J\|$ and $I$ precedes $J$ in the lexicographic ordering on $\mathbb{Z}^{n}$ (i.e., if $I \neq J$ and $i_{k}<j_{k}$, where $k$ is the least integer with $i_{k} \neq j_{k}$ ).

It can be shown that $\mathbb{Z}^{n}$ (with the Dixmier ordering) is order-isomorphic to $\mathbb{N}$; thus if $t=\sum \theta^{I} r_{I} \in T$, we can compare the $\theta^{I}$ by using the Dixmier ordering. This allows us to speak of the degree and the leading coefficient (with respect to the Dixmier ordering) of an element of $T$. Furthermore, if $X$ is any subset of $T$ then the subset of $\mathbb{Z}^{n}$ consisting of the degrees of elements of $X$ has minimal elements. If the Dixmier ordering is restricted to $\mathbb{N}^{n} \subseteq \mathbb{Z}^{n}$ then similar comments apply to the ring $T^{+}$. 
3.7. For the first part of the next theorem we temporarily relax the requirement that the ring $R$ be commutative.

Theorem. Suppose that $k$ is an algebraically closed field and that the action of $G$ on $R$ is locally finite-dimensional.

(i) If $A, B \in \mathscr{L}_{G}\left(T^{+}\right)$with $B<A$ then there exists $a \in A \backslash B$ such that $a+B$ is a $G$-eigenvector in $T^{+} / B$ and $a T^{+}+B \in \mathscr{L}_{G}\left(T^{+}\right)$.

(ii) If $R$ is commutative and $A, B \unlhd_{G} T^{+}$with $B<A$ then the element a above can be chosen to be normal modulo $B$; thus $T^{+}$(and hence $T$ ) is a polynormal ring.

Proof. Let $I$ be the minimal degree (with respect to the Dixmier ordering) of elements of $A \backslash B$, say

$$
c=\theta^{I} \gamma+\cdots \in A \backslash B
$$

where “..." denotes terms of degree lower than $I$. Put

$$
A^{0}=\{\text { leading coefficients of elements of } A \text { of degree } I\} \cup\{0\}
$$

and

$$
B^{0}=\{\text { leading coefficients of elements of } B \text { of degree } I\} \cup\{0\} \text {. }
$$

It is easily seen that $A^{0}$ and $B^{0}$ are $G$-stable right ideals of $R$ with $B^{0} \subseteq A^{0}$ and $\gamma \in A^{0} \backslash B^{0}$. Since the action of $G$ on $R$ is locally finite-dimensional, there exists a $G$-stable finite-dimensional $k$-subspace, $V$, say, of $R$ which contains $\gamma$; replacing $V$ by $V \cap A^{0}$ we may assume that $V \subseteq A^{0}$.

Since $G$ is a finitely generated abelian subgroup of $\operatorname{End}_{k} V,\left(V+B^{0}\right) / B^{0}$ contains a nonzero $G$-eigenvector $\alpha+B^{0}$, with $\alpha \in V$, by Lemma 3.4; thus there exist $\lambda_{j} \in k$ with

$$
\sigma_{j}(\alpha)-\lambda_{j} \alpha \in B^{0} \quad \forall j
$$

Note that $\lambda_{j} \neq 0$; otherwise we would have $\sigma_{j} \alpha \in B^{0}$ and then $\alpha \in B^{0}$. Since $\alpha \in A^{0}$ there exists some $a \in A \backslash B$ with $a=\theta^{I} \alpha+\cdots$. We have

$$
\sigma_{j}(a)-\lambda_{j} a=\theta^{I}\left(\sigma_{j}(\alpha)-\lambda_{j} \alpha\right)+\cdots
$$

Now by (1), $\sigma_{j}(\alpha)-\lambda_{j} \alpha \in B^{0}$, and hence there exists $b_{j} \in B$ with

$$
b_{j}=\theta^{I}\left(\sigma_{j}(\alpha)-\lambda_{j} \alpha\right)+\cdots
$$

Now $\sigma_{j}(a)-\lambda_{j} a-b_{j}$ lies in $A$ : combining (2) and (3) we see that this element has degree less than $I$ and hence must lie in $B$, so that 


$$
\sigma_{j}(a)-\lambda_{j} a \in B \quad \forall j
$$

which is to say that $a+B$ is a $G$-eigenvector in $T^{+} / B$.

Clearly $a T^{+}+B$ is a right ideal of $T^{+}$, and $\sigma_{j}\left(a T^{+}+B\right)=\sigma_{j}(a) T^{+}+B=\lambda_{j} a T^{+}+B=$ $a T^{+}+B$, so $a T^{+}+B \in \mathscr{L}_{G}\left(T^{+}\right)$.

(ii) Now suppose that $R$ is commutative and that $B<A \unlhd_{G} T^{+}$; we claim that the element $a$ provided by (i) is normal modulo $B$. Firstly,

$$
\alpha \theta_{j}=\theta_{j} \sigma_{j}(a) \equiv \theta_{j} \lambda_{j} a \quad(\operatorname{modulo} B) \quad \forall j .
$$

Also, if $r \in R$, then $A$ contains

$$
\begin{aligned}
r a-a \sigma^{I}(r) & =r\left(\theta^{I} \alpha+\cdots\right)-\left(\theta^{I} \alpha+\cdots\right) \sigma^{I}(r) \\
& =\left(\theta^{I} \sigma^{I}(r) \alpha+\cdots\right)-\left(\theta^{I} \alpha \sigma^{I}(r)+\cdots\right),
\end{aligned}
$$

which has degree less than $I$ (note that $\sigma^{I}(r) \alpha-\alpha \sigma^{I}(r)=0$ since we have assumed that $R$ is commutative), so that

$$
r a-a \sigma^{I}(r) \in B \quad \forall r \in R
$$

Together, (4) and (5) show that $a$ is normal modulo $B$, as claimed.

Theorem 3.8. If $k$ is an algebraically closed field and the action of $G$ on $R$ is locally finite-dimensional, then given $A, B \unlhd T$ with $B<A$ there exists $a \in A \backslash B$ such that $a$ is normal modulo $B$; moreover, a can be chosen to centralise $R$ modulo $B$.

Proof. Let $B^{+}=B \cap T^{+}$and $A^{+}=A \cap T^{+}$, so that $B^{+}<A^{+} \unlhd_{G} T^{+}$. Construct $a^{+}=\theta^{I} \alpha+\cdots \in A^{+} \backslash B^{+}$(where $\alpha \in R$ ) as in the proof of Theorem 3.7, so that $a^{+}$ satisfies

$$
\alpha^{+} \theta_{j}-\theta_{j} \lambda_{j} a^{+} \in B^{+} \quad \forall j
$$

(where $\lambda_{j} \in k$ ) and

$$
r a^{+}-\alpha^{+} \sigma^{I}(r) \in B^{+} \quad \forall r \in R
$$

Let $a=\theta^{-I} a^{+} \in A \backslash B$; if $r \in R$, (2) shows that $r a-a r=r \cdot \theta^{-I} a^{+}-\theta^{-1} a^{+} r=$ $\theta^{-I}\left(\sigma^{-I}(r) a^{+}-a^{+} r\right) \in \theta^{-I} B^{+} \subseteq B$. Thus $[R, a] \subseteq B$. Also,

$$
\begin{aligned}
a \theta_{j} & =\theta^{-I} a^{+} \theta_{j} \\
& \equiv \theta^{-I} \theta_{j} \lambda_{j} a^{+}\left(\operatorname{modulo} B^{+}\right), \text {by }(1)
\end{aligned}
$$




$$
\begin{aligned}
& =\lambda_{j} \theta_{j} \theta^{-I} a^{+} \\
& =\lambda_{j} \theta_{j} a .
\end{aligned}
$$

Thus $a \in A \backslash B$ is normal modulo $B$.

Definition 3.9. The ring $T=R\left[\theta_{1}, \theta_{1}^{-1}, \theta_{2}, \theta_{2}^{-1}, \ldots, \theta_{n}, \theta_{n}^{-1} ; \sigma_{1}, \sigma_{2}, \ldots, \sigma_{n}\right]$, will be said to be $R$-normally separated if, given prime ideals $P, Q \in \operatorname{Spec} T$ with $P<Q$, there exists $a \in Q \backslash P$ such that

(i) $a$ is normal (and hence a non-zero-divisor) modulo $P$.

(ii) $[R, a] \subseteq P$.

Lemma 3.10. $T$ is R-normally separated in either of the following cases:

(i) $k$ is an algebraically closed field and the action of $G$ on $R$ is locally finite-dimensional.

(ii) $k=\mathbb{Z}$ and $G$ is torsion (equivalently, finite).

Proof. (i) is immediate from Theorem 3.8 .

In case (ii), $R$ is an arbitrary commutative ring. Since $G$ is torsion, there exist $k_{j} \in \mathbb{Z} \backslash 0$ with $\sigma_{j}^{k_{j}}=1$. This means that $\theta_{j}^{k_{j}} \in Z(T)$, the centre of $T$, and so $T$ is a finite normalising extension of the commutative ring $R\left[\theta_{1}^{k_{1}}, \ldots, \theta_{n}^{k_{n}}\right]$, and so $T$ is a polynomial identity ring and hence centrally separated (see $[8,13.1 .13$ and 13.6.4]). This clearly implies that $T$ is $R$-normally separated.

3.11. We translate the property of $R$-normal separation to $S$.

Lemma. Suppose that $T$ is $R$-normally separated. If $I, J \in \operatorname{Spec}^{\Gamma} S$ and $I<J$ then there exists $c \in J \backslash I$ such that

(i) $c S+I \unlhd_{\Gamma} S$

(ii) $c$ is regular modulo $I$.

Proof. Let $P=\phi^{-1} I$ and $Q=\phi^{-1} J$. Then $P$ and $Q$ are prime ideals of $T$ with $P<Q$ and hence there exists $a \in Q \backslash P$ with $a$ normal modulo $P$ and $[R, a] \subseteq P$; note that $T a+P \unlhd T$. By Lemma 2.4, $\phi(t a)-\phi(t) \phi(a) \in I$ for all $t \in T$. Thus $\phi(T a+P)=\phi(T) \phi(a)+I$ $=S \phi(a)+I$ is a $\Gamma$-stable ideal of $S$ and $\phi(t) \cdot \phi(a) \in I \Rightarrow \phi(t a) \in I \Rightarrow t a \in p \Rightarrow t \in p \Rightarrow \phi(t) \in I$, so that $c=\phi(a)$ is regular modulo $I$, as required.

\section{Catenarity}

4.1. In this section we will show that in certain circumstances the ring $T$ is catenary. We will do this by showing that the lattice of $\Gamma$-prime ideals of $S$ is catenary. In order 
for the notion of "height" to make sense we assume from now on that $R$ is a (commutative) Noetherian ring. Recall from 1.1 that this means that both $T$ and $S$ are also Noetherian rings.

We will have to deal with several different varieties of catenarity simultaneously, and it will be convenient to describe these in an abstract framework.

Definition. Let $\Lambda$ be a partially ordered set. If $\lambda \geqq \kappa$ are elements of $\Lambda$ then a descending chain of length $t$ between $\lambda$ and $\kappa$ is a sequence $\left(\lambda_{t}, \ldots, \lambda_{0}\right)$ of elements of $\Lambda$ with $\lambda=\lambda_{t}>\lambda_{t-1}>\cdots>\lambda_{0}=\kappa$; this chain is saturated if for each $i$ there does not exist $\rho \in \Lambda$ with $\lambda_{i}>\rho>\lambda_{i-1}$. If $\lambda \in \Lambda$ then the height of $\lambda$, ht $\lambda$, is the supremum of the lengths of chains descending from $\lambda$ : it is possible for an element to be of infinite height.

Now supose that $\Lambda$ is a partially ordered set in which every element is of finite height. It is clear that $\Lambda$ satisfies the descending chain condition and that for every element $\lambda$ of $\Lambda$ there exists a minimal element $\mu$ of $\Lambda$ (i.e., an element of height zero) such that $\lambda \geqq \mu$. We denote the set of minimal elements of $\Lambda$ by Min $\Lambda$. The partially ordered set $\Lambda$ is catenary if, whenever $\lambda \geqq \kappa$ are elements of $\Lambda$, all saturated chains between $\lambda$ and $\kappa$ are of the same length (depending on the choice of $\lambda$ and $\kappa$ ). If $X$ is a subset of $\Lambda$ then we say that $\Lambda$ is uniformly catenary over $X$ if, whenever $\lambda \in \Lambda$, all descending chains from $\lambda$ to some element $\chi$ of $X$ are of the same length, irrespective of the choice of the element $\chi$. from $X . \Lambda$ is uniformly catenary if it is uniformly catenary over $\operatorname{Min} \Lambda$. It is easily seen that $\Lambda$ is uniformly catenary if and only if, whenever $\lambda \in \Lambda$ and $\mu \in \operatorname{Min} \Lambda$ with $\lambda \geqq \mu$, the length of any saturated chain between $\lambda$ and $\mu$ is equal to the height of $\lambda$.

We will apply the foregoing terminology to partially ordered sets consisting of ideals in rings. For instance, a ring $A$ will be said to be (uniformly) catenary if the partially ordered set $\operatorname{Spec} A$ is (uniformly) catenary, and if $P \in \operatorname{Spec} A$ then the height of $P$ in $A$, $\mathrm{ht}_{A} P$, is the height of $P$ when considered as an element of the partially ordered set Spec $A$. Similarly, if $\Gamma$ is some set of endomorphisms of $A$, then $A$ is $\Gamma$-catenary if the partially ordered set $\operatorname{Spec}^{\Gamma} A$ is catenary, and if $Q \in \operatorname{Spec}^{\Gamma} A$ then the $\Gamma$-height of $Q$,

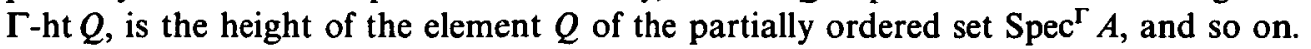

The classical Krull dimension of a ring $A$ is

$$
\text { Cl.Kdim } A=\sup \{\text { ht } P: P \in \mathrm{Spec} A\} \text {. }
$$

The adjective "classical" here is used to distinguish this dimension from the Krull dimension in the sense of Gabriel and Rentschler.

Lemma 4.2. Let $\Lambda$ be a partially ordered set in which every element has finite height. Then $\Lambda$ is catenary if and only if $\Lambda$ is uniformly catenary over $\{\mu\}$ for every $\mu \in \operatorname{Min} \Lambda$. In particular, if $\Lambda$ is uniformly catenary then $\Lambda$ is catenary.

Proof. It is clear that if $\Lambda$ is catenary then it is uniformly catenary over $\{\mu\}$ for each $\mu \in \operatorname{Min} \Lambda$. Conversely, suppose that $\Lambda$ is uniformly catenary over $\{\mu\}$ for each $\mu \in \operatorname{Min} \Lambda$. Choose elements $\lambda \geqq \kappa$ and fix $\mu \in \operatorname{Min} \Lambda$ with $\kappa \geqq \mu$. Let $\lambda=\lambda_{s}>\lambda_{s-1}>\cdots>\lambda_{0}=\kappa$ and 
$\lambda=\rho_{t}>\rho_{t-1}>\cdots>\rho_{0}=\kappa$ be saturated chains between $\lambda$ and $\kappa$. Using the descending chain condition for elements of $\Lambda$, construct a saturated chain $\kappa=\kappa_{r}>\cdots>\kappa_{0}=\mu$. We now have two saturated chains $\lambda=\lambda_{s}>\lambda_{s-1}>\cdots>\lambda_{0}=\kappa_{r}>\cdots>\kappa_{0}=\mu$ and $\lambda=\rho_{t}>$ $\rho_{t-1}>\cdots>\rho_{0}=\kappa_{r}>\cdots>\kappa_{0}=\mu$, and since $\Lambda$ is uniformly catenary over $\{\mu\}$ we have $s+r=t+r$, and so $s=t$. Thus $\Lambda$ is catenary.

Lemma 4.3. Let $\Lambda$ be a partially ordered set in which every element has finite height. Then the following are equivalent:

(i) $\Lambda$ is uniformly catenary.

(ii) If $\lambda \geqq \kappa$ are elements of $\Lambda$ with $\mathrm{ht} \lambda / \kappa=1$ then $\mathrm{ht} \lambda=\mathrm{ht} \kappa+1$, where ht $\lambda / \kappa$ denotes the height of (the image of) the element $\lambda$ in the partially ordered set $\Lambda / \kappa=\{\rho \in \Lambda: \rho \geqq \kappa\}$.

Proof. The proof is similar to that of Lemma 4.2 .

4.4. We now give some examples of types of commutative rings which are (uniformly) catenary.

Examples. (i) If $A$ is a caternary domain than it is clear that $A$ is uniformly catenary.

(ii) Suppose that $A$ is a commutative Cohen-Macaulay ring: then $A$ is catenary by [6, Corollary VI.3.15]. If $Q \in \operatorname{Spec} A$ then $A_{Q}$ is a local Cohen-Macaulay ring, so that Cl.Kdim $A_{Q}=$ Cl.Kdim $\left(A_{Q} / B\right)=\mathrm{ht}_{A} Q$ for all minimal prime ideals $B$ of $A_{Q}$ (see [6, p. 188]). Thus if $Q=Q_{t}>Q_{t-1}>\cdots>Q_{0}=P$ is a saturated chain of prime ideals with $P$ minimal, we have $\mathrm{ht}_{A} Q=\mathrm{Cl} . \mathrm{Kdim}\left(A_{Q} / P_{Q}\right)=t$ (since $A$ is catenary). Thus $A$ is uniformly catenary.

(iii) Suppose that $A$ is an affine algebra over a field $k$, and that $G \leqq A^{2} t_{k} A$ is a group of $k$-automorphisms of $A$. Let $Q \in \operatorname{Spec}^{G} A$ and let $X$ be the set of prime ideals minimal over $Q$, so that $Q=P^{G}$ for every $P \in X$. We claim that $A$ is uniformly catenary over $X$. If $B \in \operatorname{Spec} A$ and $P \in X$ then [6, Corollary II.3.5] shows that any saturated chain between $B$ and $P$ is of length $\mathrm{Cl} . \mathrm{Kdim} A / P-\mathrm{Cl} . \mathrm{Kdim} A / B$; this is clearly independent of the choice of the chain, so that $A$ is catenary. Now if $P^{\prime}$ is another element of $X$ then $P^{\prime}=P^{g}$ for some $g \in G$ and so $A / P \cong A / P^{\prime}$ via $a+P \mapsto a^{g}+P^{g}$. It follows that any saturated chain of prime ideals from $B$ to $P^{\prime}$ is of length $\mathrm{Cl} . \mathrm{Kdim} A / P^{\prime}-\mathrm{Cl} \cdot \mathrm{Kdim} A / B=$ Cl.Kdim $A / P-C l . K \operatorname{dim} A / B$ : this quantity is therefore independent of the choice of $P \in X$ and so $A$ is uniformly catenary over $X$.

4.5. In order to use the results above we need some information about $\Gamma$-primes and $G$-primes of $S$.

Lemma. (i) If $I \unlhd R$ then $S I \unlhd S$ and $S / S I \cong(R / I)\left[x_{1}^{ \pm 1}, \ldots, x_{n}^{ \pm 1}\right]$.

(ii) If $J \unlhd_{G} S$ then $J \cap R \unlhd_{G} R$. 
(iii) If $I \unlhd_{G} R$ then $S I \unlhd_{G} S$.

(iv) If $Q \in \operatorname{Spec}^{G} S$ then $Q \cap R \in \operatorname{Spec}^{G} R$.

(v) If $P \in \operatorname{Spec}^{G} R$ then $S Q \in \operatorname{Spec}^{G} S$.

Proof. (i)-(iv) can be proved by standard methods.

(v) Suppose that $Q \in \operatorname{Spec}^{G} R$ : then $S Q \unlhd_{G} S$ and $S / S Q \cong(R / Q)\left[x_{1}^{ \pm 1}, \ldots, x_{n}^{ \pm 1}\right]$. Thus it suffices to prove that if $R$ is a $G$-prime ring then so is $S$. So suppose that $R$ is $G$-prime and that $A$ and $B$ are $G$-ideals of $S$ with $A B=0$. Let $A^{\prime}$ be the set of elements of $R$ which occur as leading coefficients of elements of $A$ (with respect to the Dixmier ordering on $\mathbb{Z}^{n}$ ) and similarly let $B^{\prime}$ consist of leading coefficients of elements of $B$. Clearly $A^{\prime}$ and $B^{\prime}$ are $G$-stable ideals of $R$ and $A^{\prime} B^{\prime}=0$. Since $R$ is a $G$-prime ring we have $A^{\prime}=0$ or $B^{\prime}=0$, and this clearly implies that $A=0$ or $B=0$.

Corollary 4.6. (i) If $Q \in \operatorname{Spec}^{\Gamma} S$ then $Q \cap R \in \operatorname{Spec}^{G} R$.

(ii) If $P \in \operatorname{Spec}^{G} R$ then $S P \in \operatorname{Spec}^{\Gamma} S$; in fact, $S P$ is a $\Gamma$-stable G-prime ideal of $S$.

Proof. Firstly, note that if $I$ is any ideal of $R$ then $\chi_{r}(S I)=\chi_{r}(S) I \subseteq S I$ for every $r \in R$. Thus if $I$ is a $G$-ideal of $R$ then $S I$ is in fact a $\Gamma$-ideal of $S$ (c.f. 4.5 (iii)).

(i) Suppose that $Q \in \operatorname{Spec}^{\Gamma} S$ and that $A$ and $B$ are $G$-ideals of $R$ with $A B \subseteq Q \cap R$. We have $S A . S B \subseteq Q$, and since we have just observed that $S A$ and $S B$ are $\Gamma$-ideals of $S$ we have $A \subseteq Q \cap R$ or $B \subseteq Q \cap R$. Thus $Q \cap R$ is $G$-prime.

(ii) If $P \in \operatorname{Spec}^{G} R$ then 4.5 (iv) shows that $S P \in \operatorname{Spec}^{G} S$; however, we know that $S P$ is $\Gamma$-stable, and it is clear that $\Gamma$-stable $G$-primes are $\Gamma$-prime.

Corollary 4.7. $\operatorname{Min} \operatorname{Spec}^{\Gamma} S$ coincides with $\operatorname{Min} \operatorname{Spec}^{G} S$.

Proof. Suppose that $Q$ is a minimal $\Gamma$-prime of $S$; (i) and (ii) of 4.6 show that $Q \cap R \in \operatorname{Spec}^{G} R$ and that $S(Q \cap R)$ is a $\Gamma$-stable $G$-prime of $S$. Since $S(Q \cap R)$ is a $\Gamma$-prime contained in the minimal $\Gamma$-prime $Q$ we must have $Q=S(Q \cap R)$; thus $Q$ is a $\Gamma$ stable $G$-prime of $R$.

A similar argument using 4.5(iv) and 4.6(ii) shows that if $P$ is any minimal $G$-prime of $S$ then $P=S(P \cap R)$ is also a $\Gamma$-stable $G$-prime.

Now suppose again that $Q$ is a minimal $\Gamma$-prime of $S$, so that $Q$ is also $G$-prime. If $Q$ contains some $G$-prime $P$ then $S(P \cap R)$ is $\Gamma$-prime by $4.5(\mathrm{iv})$ and $4.6(\mathrm{ii})$, and the minimiality of $Q$ forces $Q=P=S(P \cap R)$. Thus every minimal $\Gamma$-prime of $S$ is not only $G$-prime, but is in fact minimal in the set of $G$-primes of $S$.

Using (i) and (ii) of 4.6, a similar argument shows that every minimal $G$-prime of $S$ is a minimal $\Gamma$-prime of $S$, and the results follows.

4.8. To be able to use Corollary 4.7 and the results in 4.1 and 4.2 , we must show that all elements of the sets $\operatorname{Spec}^{G} S$ and $\operatorname{Spec}^{\Gamma} S$ are of finite height. 
Lemma. Every G-prime ideal of $S$ has finite G-height.

Proof. It follows from Theorem 1.3 that there is an epimorphism of lattices Spec $S \rightarrow \operatorname{Spec}^{G} S$ given by $P \mapsto P^{G}$. Since every prime ideal in the commutative Noetherian ring $S$ is of finite height (see e.g. [1, Corollary 11.12]), it follows that every $G$-prime ideal of $S$ is of finite $G$-height.

Lemma 4.9. Every prime ideal of $T$ has finite height and hence every $\Gamma$-prime ideal of $S$ has finite $\Gamma$-height.

Proof. We will actually prove the following more general statement:

Let $A$ be a Noetherian ring in which every prime ideal has finite height. If $\sigma \in$ Aut $A$ and $B=A\left[\theta, \theta^{-1}\right]$ then $B$ is also a Noetherian ring in which every prime ideal has finite height.

The lemma will then follow because we can regard $T$ as an iterated skew Laurent extension $T=R\left[\theta_{1}^{ \pm 1} ; \sigma_{1}\right] \cdots\left[\theta_{n}^{ \pm 1} ; \sigma_{n}\right]$, and every prime of $R$ is of finite height, as in the proof of Lemma 4.8. So let $A$ and $B$ be as in (*). Suppose that $Q \in \operatorname{Spec} B$ is of infinite height; then there exist arbitrarily long chains of primes descending from $Q$. Choose $n \in \mathbb{N}$ and fix a (possibly non-saturated) chain $P_{0}<P_{1}<\cdots<P_{2 n}=Q$ of prime ideals in $B$. It follows from $[8,10.6 .4,10.6 .6]$ that we have a chain of $\sigma$-prime ideals $P_{0} \cap R<P_{2} \cap R<\cdots<P_{2 n} \cap R=Q \cap R$. This chain is of length $n$, and since $n$ was arbitrary, $Q \cap R$ is of infinite $G$-height. The argument of Lemma 4.8 shows that this is impossible, so $(*)$ is true.

4.10. The condition of $R$-normal separation allows us to pass between the lattices $\operatorname{Spec} S$ and $\operatorname{Spec}^{\Gamma} S$ without losing too much information about height.

Lemma. Let $T$ be R-normally separated. If $I<J \in \operatorname{Spec}^{\Gamma} S$ with $\Gamma$-ht $(J / I)=1$ and $P \subseteq Q \in \operatorname{Spec} S$ are minimal over $I$ and $J$ respectively then $\mathrm{ht}(Q / P)=1$.

Proof. By Lemma 3.11 there exists $c \in J \backslash I$ such that $c$ is regular modulo $I$ and $c S+I \unlhd_{\Gamma} S$. Note that $c \notin P$ since $P$ consists of zero-divisors modulo $I$; thus by the Principal Ideal Theorem $([7$, Theorem 13.5$]$ or $[8,4.1 .11])$ it will suffice to show that $Q$ is minimal over $c S+P$. Choose $B \in \operatorname{Spec} S$ with $B \subseteq Q$ and $B$ minimal over $c S+P$, and let $E$ be the largest $\Gamma$-ideal contained in $B$ : note that $c S+P \subseteq B$ so that $c S+I \subseteq E$. By Corollary $2.10, J$ is the largest $\Gamma$-ideal contained in $Q$ and it follows that $E \subseteq J$. Now suppose that $K, L \unlhd_{\mathrm{r}} S$ and that $K L \subseteq E$. Since $E \subseteq B \in \operatorname{Spec} S$ either $K$ or $L$ must be contained in $B$; as $E$ was defined to be the largest $\Gamma$-ideal contained in $B$ we must have $K \subseteq E$ or $L \subseteq E$. Thus $E$ is a $\Gamma$-prime ideal. Since $I<E \subseteq J$ and $\Gamma$-ht $(J / I)=1$ we must have $E=J$, so that $J \subseteq B \subseteq Q$. Since $Q$ is minimal over $J$ we must have $Q=B$. Thus $Q$ is minimal over $c S+P$, as required. 
4.11. We now have enough information to enable us to prove that the $\operatorname{ring} T$ is catenary in certain circumstances. We require one more definition.

Definition. If $I \unlhd S$ then $\mathscr{M}(I)=\{P \in \operatorname{Spec} S: I \subseteq P$ and $P / I \in \operatorname{Min} \operatorname{Spec} S / I\}$, the set of prime ideals minimal over $I$.

Theorem 4.12. If $T$ is $R$-normally separated and $S$ is uniformly catenary over $\mathscr{M}(Q)$ for every $Q \in \operatorname{Min} \operatorname{Spec}^{\Gamma} S=\operatorname{Min} \operatorname{Spec}^{G} S$ then $S$ is $\Gamma$-catenary and hence $T$ is catenary.

Proof. By Lemma 4.2, it suffices to show that $S$ is uniformly $\Gamma$-catenary over $\{I\}$ for each minimal $\Gamma$-prime $I$. Choose a $\Gamma$-prime $J$ of $S$ and a minimal $\Gamma$-prime $I$ contained in $J$. Fix a prime ideal $Q$ minimal over $J$. Suppose that

$$
J=J_{t}>J_{t-1}>\cdots>J_{0}=I
$$

is a saturated chain of $\Gamma$-prime ideals of $S$. Using the descending chain condition for prime ideals in $S$, construct a chain of prime ideals

$$
Q=Q_{t} \geqq Q_{t-1} \geqq \cdots \geqq Q_{0}=P,
$$

say, with $Q_{i}$ minimal over $J_{i}$ for each $i$. Theorem 4.10 shows that all of the inclusions above are proper and that the chain (**) is saturated, and since $S$ is uniformly catenary over $\mathscr{M}(I)$ we have $t=\mathrm{ht}(Q / P)$, which is independent of the choice of the chain (*). It follows that $S$ is $\Gamma$-catenary, and Theorem 2.8 now shows that $T$ is catenary.

Remark. A similar proof shows that if $S$ is uniformly catenary then $T$ is also uniformly catenary.

4.13. Recall that a Noetherian ring $A$ is said to be universally catenary if every finitely-generated $A$-algebra is catenary (see $[7, \$ \$ 15.3,32]$ ).

Corollary. Let $R$ be a commutative Noetherian algebra over an algebraically closed field $k$ and let $\sigma_{1}, \ldots, \sigma_{n}$ be commuting $k$-automorphisms of $R$. If the action of each $\sigma_{j}$ on $R$ is locally finite-dimensional then the skew Laurent polynomial ring

$$
R\left[\theta_{1}, \theta_{1}^{-1}, \theta_{2}, \theta_{2}^{-1}, \ldots, \theta_{n}, \theta_{n}^{-1} ; \sigma_{1}, \sigma_{2}, \ldots, \sigma_{n}\right]
$$

is a catenary ring in any of the following circumstances:

(i) $R$ is a universally catenary domain.

(ii) $R$ is a Cohen-Macaulay ring.

(iii) $R$ is affine over $k$.

Proof. It is clear that if $R$ is either universally catenary or affine over $k$ then the same is true of $S$. If $R$ is Cohen-Macaulay then [7, Theorem 17.7] shows that $S$ is also Cohen-Macaulay. The theorem now follows from Theorem 4.12, Lemma 3.10(i) and the examples in 4.4 . 
Note. (a) In cases (i) and (ii), Example 4.4 and the remark after Theorem 4.12 show that $T$ is actually uniformly catenary.

(b) We can use (ii) of Theorem 3.10 to prove that $T$ is catenary if $G$ is finite and $R$ is a $\mathbb{Z}$-algebra which satisfies one of (i), (ii) or (iii) above. However, we have not really gained anything by this as in this case $T$ is an affine PI-ring (as pointed out in 3.10), and a well-known theorem of Schelter states that all affine PI-algebras are catenary: see $[8,13.10 .12,13.10 .13]$.

Remark 4.14. A. D. Bell and G. Sigurdsson have shown that if $R$ is the commutative polynomial ring $k[s, t, w]$ and $\sigma$ is the $k$-automorphism of $R$ given by

$$
\sigma(s)=t, \quad \sigma(t)=s+w t^{2}, \quad \sigma(w)=w,
$$

then $R\left[\theta, \theta^{-1} ; \sigma\right]$ is not catenary (see [2, Example 3.10$]$ for details). Thus the hypothesis of local finite-dimensionality cannot be omitted from Theorem 4.13.

\section{REFERENCES}

1. M. F. AtiYAh and I. G. McDonald, Introduction to Commutative Algebra (Addison-Wesley, Reading, Mass., 1969).

2. A. D. Bell and G. Sigurdsson, Catenarity and Gelfand-Kirillov dimension in Ore extensions, J. Algebra 127 (1989), 409-425.

3. K. A. Brown, K. R. Goodearl and T. H. Lenagan, Prime ideals in differential operator rings-catenarity, Trans. Amer. Math. Soc. 317 (1990), 749-772.

4. J. E. Humphreys, Linear Algebraic Groups (Graduate Texts in Mathematics 21, SpringerVerlag, New York-Heidelberg-Berlin, 1981).

5. A. V. Jategaonkar, Localization in Noetherian Rings (London Math. Soc. Lecture Note Series 98, Cambridge University Press, Cambridge, 1986).

6. E. Kunz, Introduction to Commutative Algebra and Algebraic Geometry (Birkhäuser, Boston, 1985).

7. H. Matsumura, Commutative Ring Theory (Cambridge Studies in Advanced Mathematics 8, Cambridge University Press, Cambridge, 1986).

8. J. C. McConnell and J. C. Robson, Noncommutative Noetherian Rings (Wiley series in Pure and Applied Mathematics, Wiley, 1987).

9. J. E. Roserlade, Prime ideals in group rings of polycyclic groups, Proc. Lond. Math. Soc. (3) 36 (1978), 385-447; Corrigenda: Prime ideals in group rings of polycyclic groups. Proc. London Math. Soc (3) 38 (1979), 216-18.

Department of Mathematics

UNIVERSITY OF EDINBURGH

James Clerk Maxwell Building

KING'S BUILDINGS

MAYFIELD RoAD

EDINBURGH EH9 3JZ
Present Address:

Departments of Mathematics

UNIVERSITY OF LIVERPOOL

PO Box 147

LJVERPOOL L69 3BX 